\title{
Design of Tension Control System for Automated Fibre Placement
}

\author{
Mengjuan Chen ${ }^{1,2}$, Baolin $\mathrm{Wu}^{2,{ }^{*}}$, Yao Chen ${ }^{3}$, Tianyi Luo ${ }^{3}$ and Fengyu Yang ${ }^{4}$ \\ ${ }^{1}$ Research Center of Precision Sensing and Control, Institute of Automation, Chinese Academy of \\ Sciences, Beijing 100190, China \\ ${ }^{2}$ School of Computer and Control Engineering, University of Chinese Academy of Sciences, Beijing \\ 100049, China \\ ${ }^{3}$ College of Information Science and Technology, Beijing University of Chemical Technology, Beijing \\ 100029, China \\ ${ }^{4}$ College of Mechanical and Electrical Engineering, Beijing University of Chemical Technology, Beijing \\ 100029, China
}

Keywords: Automated Fibre Placement, Composites, Taper tension, Modeling and simulation.

\begin{abstract}
Automated Fibre Placement (AFP) has been widely used in composites manufacture and the tension control system is the key to successful placement. This article designs the structure of tension system for AFP and an in-depth analysis on the process of tension control is carried out. Then, a mathematical model of the tension control system is established and a corresponding simulation experiment is conducted in the paper. Besides, in order to overcome the wrinkle problem, taper tension control strategy instead of constant tension control strategy is used, which shows a good result.
\end{abstract}

\section{Introduction}

Composite materials are now increasingly used as large and complex primary structures in the aeronautics industry, because of their advantages over traditional materials. However, their well-known manufacturing weaknesses drive many companies to develop new methods to obtain better part quality. The Automated Fibre placement (AFP) process shows great potential for manufacturing of large composite structures. An AFP machine consists of a computer controlled robotic arm with a placement head end effector that lays bands of prepreg strips onto a mould in order to construct the layup. The tension control system of AFP is the key to successful placement. Nowadays, many companies have developed advanced AFP machines. Some representative companies like Cincinnati and Ingersoll from America, CORIOLIS from France and MTORRES from Spain, all have their own tension control system.

There are two different classification ways for tension control system. On one hand, it can be divided into two types according to the way of tension feedback: based on displacement sensor and based on tension sensor; on the other hand, according to the way of tension coming into being, it also can be divided into three types: based on cylinder, based on spring with dynamo and based on dynamo [1-3].

Nowadays, most AFP machines use constant tension control system. However, during the process of actual placement, this tension control strategy will make the material on winding roller too tight inside and too loose outside. It will even result in wrinkle and affect the quality of placement. In order to solve this problem, this article has carried out in-depth analysis on AFP tension control process and put forward a control method called taper tension control.

\section{The structure design of tension control system of AFP}

The whole mechanical structure design of AFP is shown in Fig.1-Fig.5. The whole system mainly includes three parts which are slip sheet collection, tension detection and tension adjustment. In the structural model, the Magnetic Powder Brake (MPB) connects with the tow roller. The AC servo motor shaft (requirements with a high response speed, control ability, stability and no zero drifting) connects with the winding roller. The guide roller1 (the 2nd part in Fig.1) is used as stripping roller to wipe off the lining paper which is covered on the surface of the fibre bundle. The guide roller2 is called moving roller shaft because it can slide along the slider. When the tension control system begins to turn and the 
winding side has a traction effect on the fibre, the fibre is released by the unwinding roller. For the guidance of the guide roller, the fibre is pulled out. Because the traction effect coming from the traction can produce pulling force to the fibre and the MPB can provide resistance for the uncoiling roller, the tension will come into being.

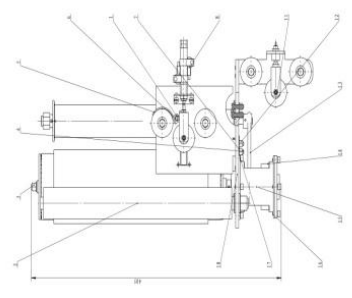

Figure1.Structure of tension control system

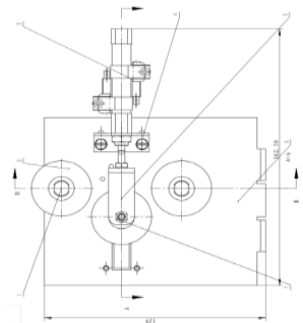

Figure 3. Tension adjustment

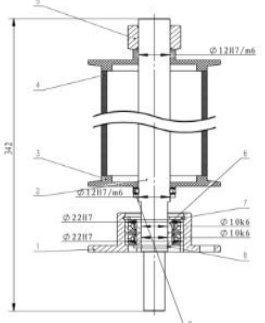

Figure 2. Slip sheet collection

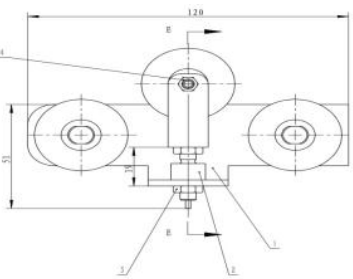

Figure4. Tension detection

\section{The modeling of the tension control system}

Fig.5 shows the schematic of this AFP tension control system.

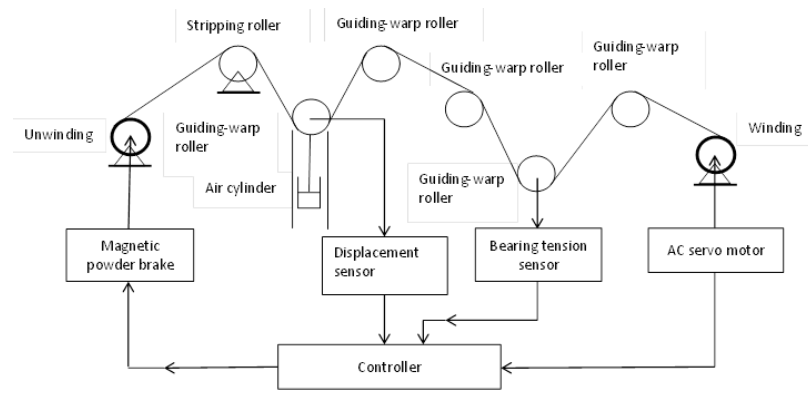

Figure 5. Tension control system

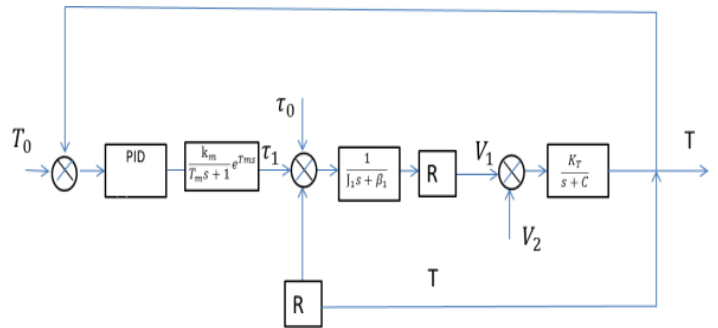

Figure 6. Control block diagram of the system

\section{A. Establishment of the mathematical model for MPB}

The growth rate of the magnetic flux of MPB depends on the inductance in excitation coil [4]. It can be approximately seen as a first order inertial loop. However, there is a time-delay process before magnetic powder in discrete state forms chain of magnetic powder, which makes MPB has hysteresis property. The open-loop transfer function can be described as [5]:

$$
G_{M}(S)=\frac{\tau_{M}(S)}{I_{M}(S)}=\frac{K_{M}}{T_{M} S+1} E^{-\tau S} .
$$

$K_{\mathrm{M}}$ is the gain of MPB; $\tau$ is the lag time of MPB; $T_{\mathrm{M}}$ is the inertia time constant.

\section{B. Establishment of the mathematical model for tension sensor of bearing type}

In general, the real-time tension needs to be detected to form the feedback of tension and a closed loop in the tension control system. Load cells are used on both unwinding side and winding side. 
The tension sensor imposes the pressure load by measuring roller to produce a small displacement of the plate as shown in the figure, which is usually within $120 \mathrm{um}$. And it detects the actual tension through differential pressure transmitter [6].

If the sensor is installed in horizontal, the left and right angles of guide roller and measuring roller are equal. And then we assume that the tension through the measuring roller is $r$, the pressure is $F$, the left and right angles are both $\theta$, the gravity of the measuring roller is $G$, and the tension of material is $T$. Through the analysis of the force of the measuring roller, it can be found:

$$
F=2 T \cos (\theta / 2)+G
$$

If the gravity of the measuring roller is ignored or the zero adjustment has been made to the sensor, the installation angle will be the only factor that can affect the relationship between the material tension and the positive pressure. Under this situation, the pressure value of the sensor is the actual value of the material tension. The transfer function can be expressed in the order of inertia [5], as shows: $\frac{\alpha}{T_{0} s+1}$

$\alpha$ is the coefficient of tension variation, $T_{0}$ is the inertial time coefficient of the sensor. However, for a large inertia system, the delay can be ignored. In addition, the delay will be converted to the controlled object. In this paper, it is regarded as a purely proportional part [5].

\section{Tension and linear velocity difference equation}

Hooke's law: Tension is caused by some external factors, which forces the material to deform, resulting in a force inside the material [2]. In the range of elastic deformation, the size of the tension is proportional to the deformation rate of the material [5].

$$
T_{1}=E A \varepsilon_{1}=E A \frac{L_{1}-L_{10}}{L_{10}}=E A \frac{\Delta L_{1}}{L_{10}}
$$

$T_{1}$ is the tension. $V_{1}$ and $\omega_{1}$ are linear velocities and angular velocities of the first rolling stand. $L_{10}$, $L_{1}$ are the length of the material which is stretched and not. $E$ and $A$ are the material of elastic modulus and the transverse area of it. $\varepsilon_{1}$ is material change rate. Then Eq. (4) and Eq. (5) can be attained:

$$
\begin{aligned}
& \Delta L_{1}=L_{1}-L_{10}=\int_{0}^{\mathrm{t}}\left(V_{2}-V_{1}\right) d t . \\
& T_{1}=\frac{E A}{L_{10}} \int_{0}^{\mathrm{t}}\left(V_{2}-V_{1}\right) d t=\frac{E A}{L_{10}} \int_{0}^{t}\left(\omega_{2} R_{2}-\omega_{1} R_{1}\right) d t .
\end{aligned}
$$

Material tension is related to linear velocity difference. In general, it is described as a first-order inertia object. Laplace transform is used to get Eq. (6):

$$
\frac{T(s)}{V_{2}(s)-V_{1}(s)}=\frac{T(s)}{\omega_{2} R_{2}-\omega_{1} R_{1}}=\frac{K_{T}}{s+C}
$$
Fig.6.

Then, a control block diagram of the system is set up by using the above principle, as shown in

The simulation parameters are shown in table1. $K_{\mathrm{p}}$ is the gain of PWM power amplifier.

According to the analysis of the tension control system, build the simulation block diagram in MATLAB as shown in Fig.7. The simulation result is shown in Fig.8.

As shown in the picture, after the parameter adjustment, the curve can rapidly track the set value. The error is also very small. The result of simulation is satisfying. 
Table 1. Simulation parameters

\begin{tabular}{|c|c|c|c|}
\hline$K_{\mathrm{r}}$ & $1.25 \times 10^{5} \mathrm{~N} / \mathrm{m}$ & $C_{12}$ & $5.6 \mathrm{~s}^{-1}$ \\
\hline$R_{1}$ & $0.3 \sim 1.2 \mathrm{~m}$ & $J_{1}$ & $1.05 \sim 2.1 \mathrm{~kg} \cdot \mathrm{m}^{2}$ \\
\hline$\beta_{1}$ & $0.024 \mathrm{~N} \cdot \mathrm{m} \cdot \mathrm{s} / \mathrm{rad}$ & $K_{\mathrm{p}} K_{\mathrm{M}}$ & $0.68 \mathrm{~N} \cdot \mathrm{m} / \mathrm{V}$ \\
\hline$T_{\mathrm{M}}$ & $0.3 \mathrm{~s}$ & $\tau_{\mathrm{M}}$ & $0.6 \mathrm{~s}$ \\
\hline
\end{tabular}

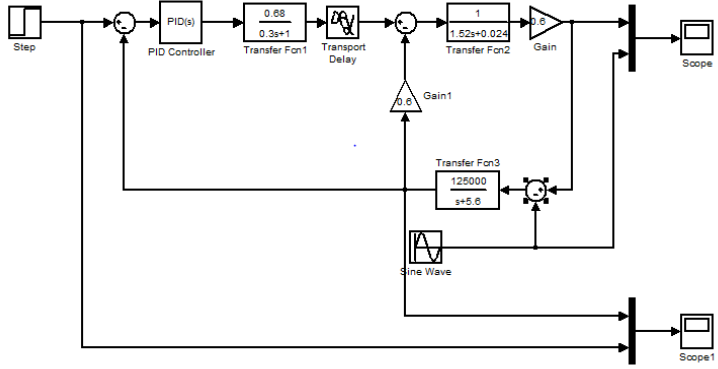

Figure 7. Simulation block diagram

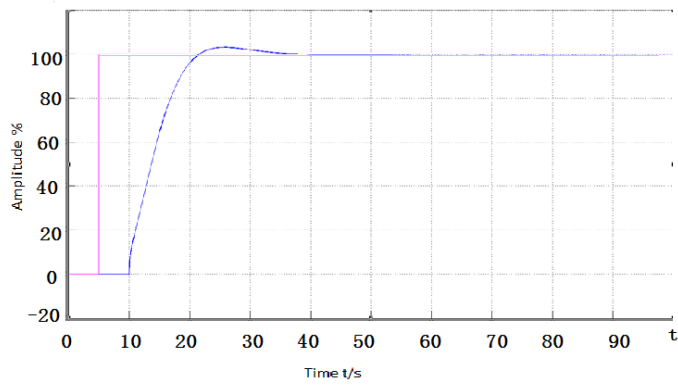

Figure 8. Simulation result

\section{The realization of taper tension control}

Constant tension control will make the material on winding roller too tight inside and too loose outside. It will even result in wrinkle and affect the quality of placement. However, taper tension control system can avoid this problem [9].

This article is based on the AFP winding system and the key to the AFP winding pre-stressed die is the taper tension control. The tension reduces as the radius of winding roller enlarges [8]. The mathematical model of taper tension control system can be described as follows [8]:

$$
T=k \cdot T_{0} \cdot f(D)
$$

$k$ is the proportionality factor; $T_{0}$ is the initial tension; $f(D)$ is the function of winding diameter.

Moment of inertia of unwinding roller consists of moment of inertia of mandrel and moment of inertia of material. The former is a fixed value and the latter is decreasing. We define the density of material $\rho$, width of material $b$, the radius of unwinding roller $R_{1}(\mathrm{t})$, the radius of mandrel $R_{0}$ and moment of inertia of material $J_{11}(\mathrm{t})$.

Moment of inertia of material:

$$
J_{11}(t)=\int_{R_{0}^{t}}^{R^{(}(t)} r^{2} d m=\int_{R_{0}^{t}}^{R^{(t)}} r^{2} \cdot \rho b \cdot 2 \pi r \cdot d r=\frac{1}{2} \pi b \rho\left(R_{1}^{4}(t)-R_{0}^{4}\right)
$$

Moment of inertia of mandrel:

$$
J_{10}=\int_{0}^{R} 0 r^{2} d m=\int_{0}^{R} 0 r^{2} \cdot \rho_{0} b \cdot 2 \pi r \cdot d r=\frac{1}{2} \pi b \rho_{0} R_{0}^{4}
$$

Moment of inertia of unwinding roller:

$$
\begin{aligned}
& J_{1}(t)=J_{10}+J_{11}(t)=\frac{1}{2} \pi b \rho_{0} R_{0}^{4}+\frac{1}{2} \pi b \rho\left(R_{1}^{4}(t)-R_{0}^{4}\right) \\
& T_{1} R_{1}=\tau_{1}+\tau_{0}+\frac{d\left(J_{1} \omega_{1}\right)}{d t}=\tau_{1}+\tau_{0}+\left(J_{1} \frac{d \omega_{1}}{d t}+\omega_{1} \frac{d J_{1}}{d t}\right)
\end{aligned}
$$


$\omega \frac{\mathrm{dJ}}{\mathrm{dt}}$ is such a small number that can be ignored.

In general, such a relatively complex model is not necessary. Using linear taper tension model can meet the requirements [8]. The taper tension control model of winding side is shown in Fig.10.
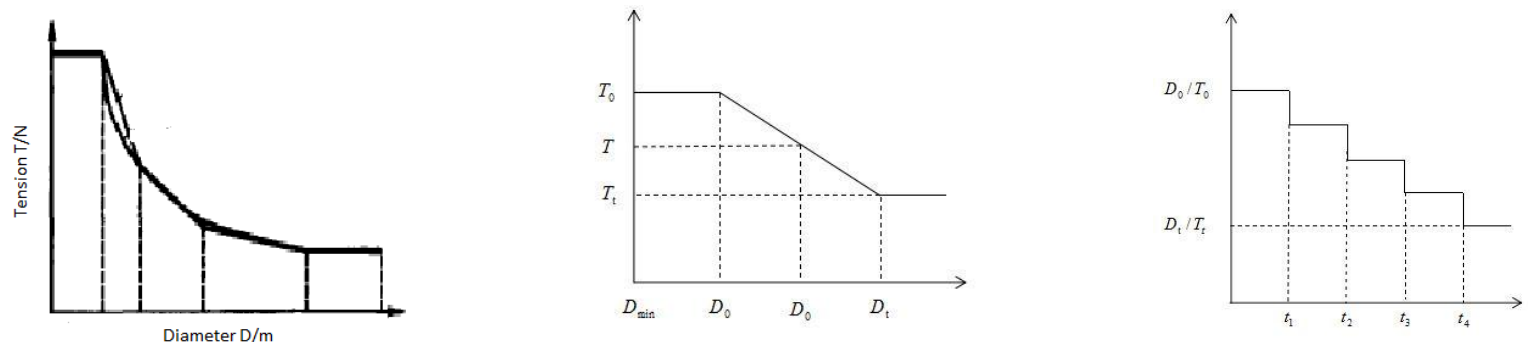

Figure9. Curve of taper tension control Figure10. Taper tension control model Figure 11. The approximate taper tension

$T_{0}$ is the initial working tension; $T_{\mathrm{t}}$ is the final working tension; $D_{0}$ is the initial diameter of taper tension control; $D_{\mathrm{t}}$ is the final diameter of taper tension control; $T$ is the tension corresponding to coil diameter $\mathrm{D}$ at any time. So, mathematical model of taper tension can be expressed as Eq. (12):

$$
T=\left\{\begin{array}{cc}
T_{0} & D<D_{\min }<D_{0} \\
T_{0}+k\left(D-D_{0}\right) & D_{j} \leq D \leq D_{i} \\
T_{i} & D_{i}<D
\end{array}\right.
$$

Where linear proportional coefficient can be expressed as Eq. (13):

$$
k=\frac{T_{i}-T_{0}}{D_{i}-D_{0}}
$$

In the actual system, the whole winding process can be divided into several periods according to the thickness of the coil and the technological requirement. The tension is constant over a period of time, but as a whole, the taper tension control system can be achieved, the tension of which decreases with time. It not only reduces the inconvenience of simulation and practical, but doesn't affect the authenticity of the system. Therefore, the model of tension, roll diameter and time can be set up.

In our model, the speed of winding side is uncontrolled, which exists as a disturbance. So, change the setting value of tension according to the unwinding diameter not the winding diameter. Due to the speed of unwinding side is constant, it is simple and accurate to change the tension based on the time. According to our model, we assume that the unwinding speed is $20 \mathrm{~m} / \mathrm{min}$, the length of tow is $100 \mathrm{~m}$, and the whole process should be completed within $5 \mathrm{~min}$. It can be assumed that the tension is constant in every $30 \mathrm{~s}$. According to the literature data [7], the tension is usually $5 \sim 20 \mathrm{~N}$. The initial tension is set as $6 \mathrm{~N}$, and the final tension is set as $10 \mathrm{~N}$. During the whole process, unwinding diameter changes from $95 \mathrm{~mm}$ to $90 \mathrm{~mm}$.

On the basis of the above parameters, a preliminary taper tension curve can be made. The formula can be represented as follows:

$$
T=\left\{\begin{array}{cc}
6 & D=90 \\
10-0.8(D-90) & 95 \mathrm{~mm}<D<95 \mathrm{~mm} \\
10 & D=95 \mathrm{~mm}
\end{array}\right.
$$

The curve of taper tension control is as Fig.12. 
Using the above curve as tension setting value, control system simulation can be built and simulation time is 300s. The trend curve of tension and time can be observed through the oscilloscope after running. Through the tension oscilloscope, the changes of tension are shown in Fig.13.

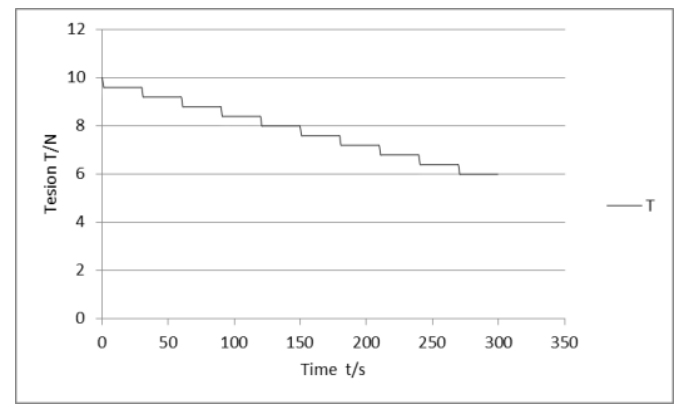

Figure 12. The curve of taper tension control

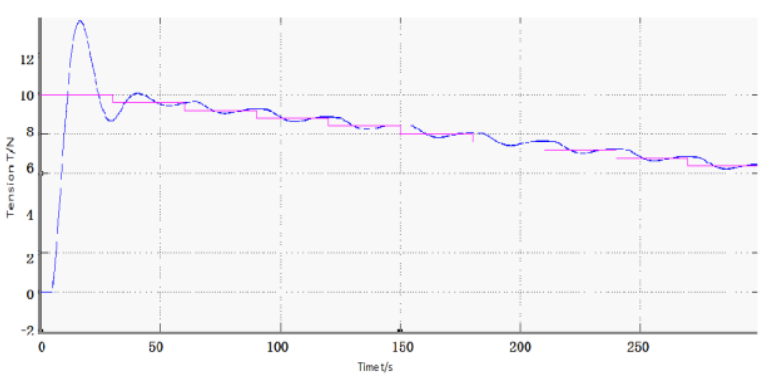

Figure 13. The simulation curve of tension

\section{Conclusion}

The tension control system of AFP is the key to successful placement. Using taper tension control to divide consist tension control into subsection control can solve the problem of crease. In this way, the quality of placement has been greatly increased.

There is still room for improvement through the tension control process. In this article, we use the taper tension to improve the quality of control. However, we can also take use of advanced control algorithms instead of PID for better control effect.

\section{References}

[1] Lopes CS, Gurdal Zafer, Camanho PP.

Variable-stiffness composite panels:buckling and first-ply failure improvements over straight-fiber laminates.Comput Struct 2008(86):897-907.

[2] Gurdal Zafer, Tatting Brian F, Wu CK, Variable stiffness composite panels: Effects of stiffness variation on the in-plane and buckling response. ComposPart A: Appl Sci Manuf 2008(5):911-22.

[3] Chauncey Wu K, Gurdal Zafer, Starnes Jr James H. Structural response of compression-loaded, tow-placed, variable stiffness panels. Foury-third AIAA structural dynamics and materials conference, Denver, Colorado; 2002. p. 22.

[4] Zhibin Wang, The tension control system development of automated fibre placement based on DSP [D].Harbin:Harbin Institute of Technology, 2013.

[5] Hengzhou $\mathrm{Yu}$,The research of compound machine tension system modeling and control strategy[D].Changsha: Central South University, 2007.

[6] Chaoyuan Chen, Modeling and simulation of multivariable tension control system[D].Changsha: Central South University, 2007.

[7] Jicheng Liu, The tension control system development of automated fibre placement[D].Harbin:Harbin Institute of Technology, 2012.

[8] Lei Yi, Guoliang Chen, Xiyan Zhang, Haifeng Yuan. The Tension Control of the Crude Foil Engine [N]. Journal of Hubei university of technology, 2009-08 (24).

[9] Jia Han.The fuzzy control of steel strip winding taper tension system [D].Jinan: Jinan University, 2014 